\title{
Efficacy of hepatitis B vaccination in patients with ulcerative colitis: a prospective cohort study
}

\author{
Anurag Mishra, Amarender Singh Puri, Sanjeev Sachdeva, Ashok Dalal \\ Department of Gastroenterology, Govind Ballabh Pant Institute of Postgraduate Medical Education and Research (GIPMER), New Delhi, India
}

Background/Aims: Response to vaccine in patients with inflammatory bowel disease is lower than in the general population. We aimed to evaluate the efficacy of hepatitis B virus (HBV) vaccination in patients with ulcerative colitis (UC) versus controls. Methods: We prospectively compared antibody response to HBV vaccination in 100 patients with UC versus controls. HBV vaccination was given to all the cases and controls at 0,1 and 6 months. Anti-hepatitis B surface (anti-HBs) titers were then measured 4 weeks after the first and the third dose. Adequate immune response (AIR) was considered if the anti-HBs titer was $>10 \mathrm{IU} / \mathrm{L}$ and effective immune response (EIR) if the anti-HBs titer was $>100 \mathrm{IU} / \mathrm{L}$. Results: Median anti-HBs titer was lower in patients with UC than controls ( $67 \mathrm{IU} / \mathrm{L}$ vs. $105 \mathrm{IU} / \mathrm{L}, P<0.01$ ). AIR and EIR were significantly lower in patients than in controls ( $82 \%$ vs. $96 \%, P=0.001 ; 41 \%$ vs. $66 \%, P<0.001$, respectively). Univariate analysis showed that age $<30$ years, mild to moderate severity of disease, disease duration $<5$ years, male sex, post first dose anti-HBs titer $>2 \mathrm{IU} / \mathrm{L}$ and non-exposure to corticosteroids, azathioprine and biologicals were predictors of AIR in patients with UC $(P<0.05)$. Multivariate analysis revealed that only nonexposure to corticosteroids, azathioprine and biologicals, male sex, and disease duration $<5$ years were independent predictors of AIR. Conclusions: Response rate to the HBV vaccination in patients with UC was significantly lower as compared to the controls. Male sex, shorter disease duration, and non-exposure to immunomodulators were independent predictors of AIR. (Intest Res 2022;20:445-451)

Key Words: Colitis, ulcerative; Hepatitis B; Vaccination; Adequate immune response; Effective immune response

\section{INTRODUCTION}

Patients with inflammatory bowel disease (IBD) have an increased risk of hepatitis B virus (HBV) infection due to malnutrition, blood transfusion, frequent use of immunosuppressants and procedures (endoscopic/surgical), which are usually required during the course of the disease. ${ }^{1}$ According to one study almost half of the IBD patients would have at least one risk factor of hepatitis B transmission. ${ }^{2}$ The mainstay of prevention of $\mathrm{HBV}$ and consequent acute and chronic liver disease is HBV vaccination. ${ }^{3,4}$ The response to the vaccine in the

Received July 29, 2021. Revised September 30, 2021.

Accepted October 8, 2021.

Correspondence to Amarender Singh Puri, Department of Gastroenterology,

GIPMER, New Delhi 110002, India. Tel: +91-11-23234242, Fax: +91-11-

23221835,E-mail: amarender.puri@gmail.com patients of IBD seems to be considerably lower than in the general population, probably because of the aforementioned factors. ${ }^{1-5}$ In healthy subjects nonresponse to the vaccination has been associated with multiple factors such as smoking, genetic factors, and advanced age. ${ }^{6}$ According to the World Health Organization (WHO), anti-hepatitis B surface (antiHBs) titer concentration $\geq 10 \mathrm{IU} / \mathrm{L}$ is considered a reliable marker of protection against HBV infection. ${ }^{7}$ Among immunocompromised patients who respond to the vaccine, clinical HBV infection has been documented in those who do not maintain an anti-HBs titer $\geq 10 \mathrm{IU} / \mathrm{L}$. India falls in the intermediate endemicity zone for hepatitis B with a prevalence ranging between $2 \%$ and $7 \%$ and a disease burden of nearly 50 million. ${ }^{8}$ However, there is no prospective study from India on the response of IBD patients to HBV vaccination. The aim of the present study was therefore to evaluate the efficacy and 
immunogenicity of the HBV vaccine in a cohort of patients with ulcerative colitis (UC) versus control subjects. To maintain homogeneity, we excluded patients with Crohn's disease (CD) as the latter is much less prevalent and there is diagnostic confusion between tuberculosis and CD.

\section{METHODS}

\section{Study}

This prospective cohort study was conducted in the department of gastroenterology, between October 2018 to March 2020. We conducted this study in compliance with the principles of the Declaration of Helsinki. The study's protocol was reviewed and approved by the Institutional Review Board of Maulana Azad Medical College (IRB No. MAMC/2018/55). Written informed consent was obtained from all the study subjects. One hundred patients of IBD (UC) with no prior history of $\mathrm{HBV}$ vaccination or HBV related infection were taken as cases and 100 non-IBD subjects, aged between 18 and 60 years who were attending the outpatient department for dyspepsia/irritable bowel syndrome or healthy subjects were taken as controls (Fig. 1). Exclusion criteria were evidence of current or past $\mathrm{HBV}$ infection, previously vaccinated patients, pregnant females, UC with concurrent malignancy, UC with toxic megacolon or acute severe colitis, HIV (human immunodeficiency virus), chronic kidney disease, and uncontrolled di-

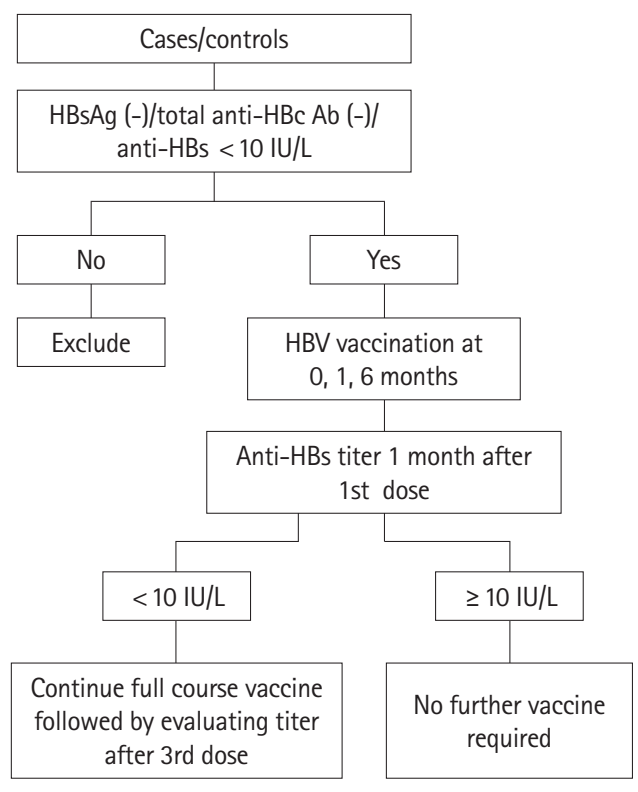

Fig. 1. Study flowchart. HBsAg, hepatitis B surface antigen; anti$\mathrm{HBC} A \mathrm{~B}$, anti-hepatitis B core antibody; anti-HBs, anti-hepatitis B surface; $H B V$, hepatitis $B$ virus. abetes mellitus. The diagnosis of UC was made on the basis of the clinical presentation supported by appropriate endoscopic, radiological, and histological investigations. Detailed evaluation was made with respect to duration of the disease, severity, extent of the disease, presence or absence of extraintestinal manifestation and exposure to corticosteroids, immunomodulators and biologicals. ${ }^{9}$ The diagnosis of dyspepsia and irritable bowel syndrome was made as per Rome IV criteria. ${ }^{10}$ Blood samples were collected before vaccination to exclude HBV infection. After the first dose of the vaccine the study subjects were telephonically contacted to revert with anti-HBs titer. Similarly, they were telephonically contacted after the third dose for evaluation of the anti-HBs titer. The subjects who did not come back after the first or the third dose were excluded from the study. Both the cases and controls were tested for hepatitis B surface antigen (HBsAg), total anti-hepatitis B core antibody (anti-HBc $\mathrm{Ab}$ ), anti-HBs titer, and liver function test. After exclusion of HBV infection, HBV vaccination (Revac-B/ Bharat biotech/purified hepatitis B type surface antigen $1 \mathrm{~mL}$ ) was injected in the deltoid to all the cases and controls as per standard WHO recommendations at 0,1 , and 6 months. ${ }^{11}$ Adequate immune response (AIR) was defined as anti-HBs titer $>10 \mathrm{IU} / \mathrm{L}$ and effective immune response (EIR) was defined as anti-HBs titer $>100 \mathrm{IU} / \mathrm{L} .^{1-7} \mathrm{~A}$ serum anti-HBs level below 10 $\mathrm{IU} / \mathrm{L}$ was counted as a nonresponder. If any case and control was lost to follow-up within 1 month after vaccination then that subject was excluded from the study.

\section{Statistical Analysis}

Data analysis was done by SPSS software version 25 (IBM Corp., Armonk, NY, USA). Continuous variables were expressed as mean and standard deviation if they were normally distributed. Median and interquartile ranges were used for continuous variables when they were not normally distributed. To compare paired nonparametric data Wilcoxon signed-rank test was used. Categorical variables were expressed as proportions and were analyzed by the chi-square test or the Fisher exact test. Continuous variables were compared by Students t-test or Mann-Whitney $U$ test where indicated. Multivariate logistic regression analysis was performed for the variables found statistically significant in the univariate analysis. A $P$-value of $<0.05$ was considered to be statistically significant.

\section{RESULTS}

The mean age of the patients in the UC group (59 males) and 
controls (54 males) was $32.8 \pm 11.4$ years and $36.0 \pm 10.3$ years respectively $(P=0.04)$. In the UC group, the proportion of patients with mild, moderate, and severe disease were 14 (14\%), 69 (69\%), and 17 (17\%), respectively. The mean duration of UC was 60.3 months. The extent of the disease was E1 (12\%), E2 (25\%), and E3 (63\%). Extraintestinal manifestations were documented in 6 patients (6\%; peripheral arthropathy in 5 and episcleritis in 1 patient). Sixty-four patients each had exposure to corticosteroids and thiopurine. None of the patients were taking steroids at the time of vaccination. Only $10 \%$ of patients had been exposed to biologicals (infliximab). Their findings are summarized in Table 1.

The median anti-HBs titer was lower in the UC group than in the controls (67 IU/L [16-202] vs. $105 \mathrm{IU} / \mathrm{L}$ [57-229], respectively, $P<0.002$ ). The proportion of patients who achieved AIR was also lower in the UC group than in the controls (82\% vs. $96 \%, P<0.001)$. EIR was also less frequent in the UC group than in the controls ( $41 \%$ vs. $66 \%, P<0.001$ ). These observations are tabulated in Table 2.

AIR was achieved in 42 of the 46 patients (91.3\%) cases with age $\leq 30$ years as compared to 40 of the 54 patients $(74.1 \%)$ in those with age $>30$ years $(P=0.036)$. Frequency of AIR in UC patients was higher in males as compared to females $(89.8 \%$

Table 1. Baseline Characteristics of Patients with Ulcerative Colitis

\begin{tabular}{lc}
\hline Characteristics & Value $(n=100)$ \\
\hline Age $(y r)$ & $32.8 \pm 11.4$ \\
Sex & $59(59)$ \\
Male & $41(41)$ \\
Female & \\
Disease severity & $14(14)$ \\
Mild & $69(69)$ \\
Moderate & $17(17)$ \\
Severe & $60.3 \pm 62.6$ \\
Disease duration (mo) & \\
Extent of disease & $12(12)$ \\
E1 & $25(25)$ \\
E2 & $63(63)$ \\
E3 & \\
Exposure to immunosuppressants & $64(64)$ \\
Corticosteroids & $64(64)$ \\
Azathioprine/6-MP & $10(10)$ \\
Biologicals & \\
\hline
\end{tabular}

Valuse are presented as mean \pm standard deviation or number (\%). 6-MP, 6-mercaptopurine. vs. $70.7 \%, P=0.014)$.

AIR was achieved in 46 of the 64 patients (72\%) of the UC patients who had history of exposure to corticosteroids as compared to 36 patients $(100 \%)(P<0.001)$ who were not exposed to corticosteroids. In patients with UC with history of thiopurine exposure, AIR was achieved in 46 of the 64 patients (72\%) as compared to 36 patients (100\%) who were not exposed to thiopurines $(P<0.001)$. In UC patients with prior history of exposure to both corticosteroid as well as azathioprine, AIR was achieved in 39 of the 57 (68.4\%) patients as compared to 43 patients $(100 \%)$ who were not exposed to this combination $(P<0.001)$. In patients with UC with history of exposure to biologicals, AIR was achieved in 5 of the 10 patients (50\%) cases as compared to 77 of the 90 patients $(85.5 \%)$ who were not treated with biologicals $(P=0.016)$.

AIR was achieved in 72 of the 83 patients (86.8\%) with mild to moderate disease activity as compared to 10 of the 17 patients $(58.8 \%)$ in those with severe disease activity $(P=0.017)$. AIR was achieved in 33 of the 37 patients (89.2\%) with limited colitis as compared to 49 of the 63 patients $(77.8 \%)$ with pancolitis $(P=0.185)$. AIR was significantly higher in the patients with $\leq 5$ years disease duration than those with duration $>5$ years $(88.2 \%$ vs. $68.8 \%, P=0.018)$. AIR was achieved in 59 of the 76 patients $(77.6 \%)$ if the post first dose titer was $\leq 2 \mathrm{lU} / \mathrm{L}$ as compared to 23 of the 24 patients (95.8\%) in whom the post first dose titer $>2 \mathrm{lU} / \mathrm{L}(P=0.034)$.

Only 5 patients in the UC group had anti-HBs titer $>10 \mathrm{IU} / \mathrm{L}$ after the first dose as compared to 15 patients in the control group.

On applying univariate analysis it was observed that age $<30$ years, mild to moderate severity of disease, disease duration $<5$ years, male sex, post first dose anti-Hbs titer $>2 \mathrm{IU} / \mathrm{L}$ and non-exposure to corticosteroid, azathioprine, and biologicals were predictors of AIR in patients with UC $(P<0.05)$. On applying multivariate analysis we found that non-exposure to

Table 2. Response to Hepatitis B Virus Vaccination in the 2 Study Groups

\begin{tabular}{lccc}
\hline Parameter & $\begin{array}{c}\text { Case group } \\
(n=100)\end{array}$ & $\begin{array}{c}\text { Control group } \\
(n=100)\end{array}$ & P-value \\
\hline Anti-HBs titer (IU/L) & $67(16-202)$ & $105(57-229)$ & 0.003 \\
AIR (anti-HBs $>10 \mathrm{IU} / \mathrm{L})$ & $82(82)$ & $96(96)$ & $<0.001$ \\
EIR (anti-HBs $>100 \mathrm{IU} / \mathrm{L})$ & $41(41)$ & $66(66)$ & $<0.001$ \\
\hline
\end{tabular}

Valuse are presented as median (interquartile range) or number (\%). anti-HBs, anti-hepatitis B surface; AIR, adequate immune response; EIR, effective immune response. 
corticosteroids, azathioprine, biologicals, male sex, and disease duration $<5$ years were all independent predictors of AIR in patients with UC (Table 3). None of the patients (UC) or the controls had any serious adverse event following the vaccination.

\section{DISCUSSION}

This is the largest prospective cohort study from Asia which compares the response of the standard 3-dose HBV vaccination in patients with IBD (UC) compared to controls. A prior prospective study from Korea of 73 patients included both vac-

Table 3. Factors Affecting Adequate Immune Response-Multivariate Analysis

\begin{tabular}{lc}
\hline Variable & $P$-value \\
\hline Sex (male vs. female) & 0.043 \\
Disease duration ( $\leq 5 \mathrm{yr}$ vs. $>5 \mathrm{yr}$ ) & 0.026 \\
Exposure to corticosteroid & $<0.001$ \\
Exposure to azathioprine & 0.001 \\
Exposure to biological & 0.023 \\
Exposure to corticosteroid and azathioprine & $<0.001$ \\
Age ( $<30$ yr vs. $>30$ yr) & 0.570 \\
Severity of disease (mild to moderate vs. severe) & 0.182 \\
Post first dose anti-HBs titer ( $\leq 2$ IU/L vs. $>2$ IU/L) & 0.082 \\
\hline
\end{tabular}

anti-HBs, anti-hepatitis B surface. cine nonresponders and vaccine naïve patients with both $\mathrm{CD}$ and UC. ${ }^{12}$ Only 29 patients in this study had the complete vaccination schedule of 3 doses which is considerably lesser than the sample size in the current study. In the current study, both AIR and EIR were significantly lower in patients with UC versus controls: $82 \%$ versus $96 \%$ and $41 \%$ versus $66 \%$, respectively. The current study shows that female sex, disease duration $>5$ years and exposure to corticosteroids, thiopurines and biologicals are independent predictors of poor response to the standard 3 dose vaccination in patients with UC.

Till now a total of 11 studies both prospective and retrospective have evaluated the response of HBV vaccination in IBD patients. Of these only 4 studies were prospective in design. Each of these 4 studies enrolled patients with both UC and CD, whereas our study was focused only on patients with UC. In these studies, the response rate of $\mathrm{HBV}$ vaccination varied from $34 \%$ to $89 \%$. There is a near unanimous agreement on the fact that patients with IBD have a lower response rate to the standard 3 dose vaccination strategy as compared to the general population or non-IBD controls (Table 4). The vaccine response rate in the current study of $82 \%$ is well within this range.

Our findings are different from the published literature on 2 accounts. First, we showed that males had a higher response rate as compared to females. However, in the meta-analysis done by Jiang et al. ${ }^{13}$ which evaluated 13 studies showed there was no sex differences in the response rate of vaccination. The

Table 4. Response Rate of HBV Vaccination in Different Studies

\begin{tabular}{|c|c|c|c|c|c|}
\hline \multirow{2}{*}{ Author (year) } & \multirow{2}{*}{ Country } & \multirow{2}{*}{ Study type } & \multicolumn{2}{|c|}{ No. of IBD patients } & \multirow{2}{*}{$\begin{array}{l}\text { HBV vaccine response } \\
(\%)\end{array}$} \\
\hline & & & UC & $C D$ & \\
\hline Vida Pérez et al. (2009) & Spain & Retrospective & 45 & 55 & 34 \\
\hline Altunöz et al. (2012) ${ }^{1}$ & Turkey & Prospective & 62 & 38 & 76 \\
\hline Gisbert et al. $(2012)^{15}$ & Spain & Prospective & 31 & 69 & 60 \\
\hline Sempere et al. $(2013)^{16}$ & Spain & Retrospective & 24 & 76 & 48 \\
\hline Andrade et al. (2015) ${ }^{17}$ & Portugal & Prospective & 21 & 79 & 76 \\
\hline Belle et al. $(2015)^{18}$ & France & Retrospective & 20 & 80 & 79 \\
\hline Cekic et al. $(2015)^{19}$ & Turkey & Retrospective & 52 & 48 & 57 \\
\hline Cossio-Gil et al. $(2015)^{20}$ & Spain & Retrospective & 59 & 110 & 51 \\
\hline Etzion et al. $(2016)^{21}$ & Israel & $\mathrm{RCT}$ & 21 & 79 & 75 \\
\hline Jiang et al. $(2017)^{13}$ & China & Meta-analysis & 426 & 732 & 61 \\
\hline Chang et al. (2018) ${ }^{12}$ & Korea & Prospective & 28 & 45 & 89 \\
\hline Pratt et al. $(2018)^{22}$ & USA & Retrospective & 137 & 246 & 55 \\
\hline Current study (2020) & India & Prospective & 100 & 0 & 82 \\
\hline
\end{tabular}

$H B V$, hepatitis B virus; IBD, inflammatory bowel disease; UC, ulcerative colitis; CD, Crohn's disease; RCT, randomized controlled trial. 
same meta-analysis also showed that age younger than 26 years was associated with a higher response to the $\mathrm{HBV}$ vaccination. The variation from the latter observation in the current study could be explained by the fact that most of our patients in the UC were older as the mean age of our cohort was 32 years.

Patients on immunosuppressants have a poorer response to $\mathrm{HBV}$ vaccination. Nguyen et al. ${ }^{23}$ showed there was a $65 \%$ lower chance of achieving AIR in patients on $\geq 2$ immunosuppressant drugs. The maximum impact is seen in patients who have been exposed to anti-tumor necrosis factor (anti-TNF) drugs prior to vaccination. More recently Pratt et al. ${ }^{22}$ found that patients exposed to infliximab were significantly less likely to possess anti-HBs levels $\geq 10 \mathrm{IU} / \mathrm{L}$. In the current study, AIR was achieved in only $50 \%$ of the 10 patients who had been exposed to anti-TNF therapy which is in accordance with the meta-analysis done by Nguyen et al. ${ }^{23}$ which clearly demonstrated that patients on immunomodulators or anti-TNF monotherapy had lower response rates to $\mathrm{HBV}$ vaccination.

A unique feature of our study was the determination of the anti-HBs titer after the first dose. This was done to determine if the patients in either the study group or the controls had received HBV vaccination but did not have any record or recollection of the same. Only $5 \%$ of the study group had an anti-HBs titer $>10 \mathrm{IU} / \mathrm{L}$ suggesting an anamnestic response. The corresponding value in the control group was significantly higher $15 \%(P<0.01)$. The implication of this observation is that these patients need not be given the remaining 2 doses of the HBV vaccine.

The current study has clinical implications for all those countries like China, India, and Korea which have a high disease burden of both IBD and hepatitis B. The prevalence of hepatitis B in India ranges between $1.4 \%$ and $2.7 \%$ in the general population. ${ }^{24}$ Horizontal transmission accounts for nearly twothirds of disease burden in India. The prevalence of UC in India is in the range of $40-45$ per $100,000 .^{25}$ Thus from an Indian perspective there is a large number of patients of UC who are potentially vulnerable to hepatitis B considering that the current population of India is about 1.38 billion. In a study from North America Melmed et al. ${ }^{2}$ showed that less than $30 \%$ of IBD patients actually received the HBV vaccination despite having risk factors for acquiring hepatitis B infection. The corresponding figures for Asian countries like China and India are likely to be even lower than this. Considering the vast number of non-immunized IBD patients in these countries it would be prudent to start vaccinating the most vulnerable group, i.e., those with severe disease who are either on anti-TNF drugs or are being considered for biological therapy.

The current study has a few limitations. The post-vaccination follow-up period was limited. The moot point whether patients with a low antibody titer $(<100 \mathrm{IU} / \mathrm{L})$ are protected against $\mathrm{HBV}$ infection due to an anamnestic response remains unanswered. Only if this point is proven beyond doubt is it worthwhile to aim for an EIR value $\geq 100 \mathrm{IU} / \mathrm{L}$ in these patients. The other limitation of our study is the small number $(10 \%)$ of patients who were exposed to anti-TNF therapy. The low usage of biological therapy in India is related to the high cost of biological therapy and the lack of medical insurance coverage in most of the population. ${ }^{26}$ There is therefore a need for multicentric studies having a significantly high number of IBD patients who have been exposed to biological therapy and subsequently evaluated for the efficacy of the HBV vaccination. Smoking may affect response to HBV vaccination but in our study it was not excluded which may be considered as a limitation. However the overall smoking rates in India are amongst the lowest in Asia. As per recent data, smoking percentage in India is only $14.8 \%$ as compared to other Asian countries like Indonesia, Korea, and Jordan where it ranges between $27 \%$ and $40.9 \%{ }^{27}$ The smoking prevalence in females in Asia as per WHO data is only $2 \%$. Therefore it is unlikely that the smoking status would have had a significant effect on the outcome of our study.

In conclusion, the current study shows that response rates (both AIR and EIR) to the HBV vaccination are considerably lower in South Asian patients with UC, who are female have longer duration of disease and have been exposed to immunosuppressants especially biologicals. Therefore the HBV vaccination status should be evaluated at the time of the initial diagnosis of IBD. In those who are not immunized, HBV vaccination should be administered preferably before exposure to immunosuppressive therapy.

\section{ADDITIONAL INFORMATION}

\section{Funding Source}

The authors received no financial support for the research, authorship, and/or publication of this article.

\section{Conflict of Interest}

Puri AS is an editorial board member of the journal but was not involved in the peer reviewer selection, evaluation, or decision process of this article. No other potential conflicts of interest relevant to this article were reported. 


\section{Data Availability Statement}

Not applicable.

\section{Author Contribution}

Conceptualization: Puri AS, Mishra A. Data curation: Mishra A. Formal analysis: Puri AS, Sachdeva S, Dalal A. Investigation: Puri AS, Mishra A. Methodology: Puri AS, Mishra A. Project administration: Mishra A. Supervision: Puri AS. Validation: Sachdeva S, Dalal A. Visualization: Sachdeva S, Dalal A. Writing original draft: Puri AS, Mishra A. Writing - review \& editing: Puri AS, Sachdeva S, Dalal A. Approval of final manuscript: All authors.

\section{ORCID}

Mishra A https://orcid.org/0000-0002-2662-3703

Puri AS https://orcid.org/0000-0002-3663-2494

Sachdeva S

Dalal A https://orcid.org/0000-0002-5588-1111 https://orcid.org/0000-0001-7590-2226

\section{REFERENCES}

1. Altunöz ME, Senateș E, Yeșil A, Calhan T, Ovünç AO. Patients with inflammatory bowel disease have a lower response rate to $\mathrm{HBV}$ vaccination compared to controls. Dig Dis Sci 2012;57: 1039-1044.

2. Melmed GY, Ippoliti AF, Papadakis KA, et al. Patients with inflammatory bowel disease are at risk for vaccine-preventable illnesses. Am J Gastroenterol 2006;101:1834-1840.

3. Hou JK, Velayos F, Terrault N, Mahadevan U. Viral hepatitis and inflammatory bowel disease. Inflamm Bowel Dis 2010; 16:925-932.

4. Nguyen CT, Tran TT. Hepatitis vaccination and prophylaxis. Clin Liver Dis 2009;13:317-329.

5. Sands BE, Cuffari C, Katz J, et al. Guidelines for immunizations in patients with inflammatory bowel disease. Inflamm Bowel Dis 2004;10:677-692.

6. Fisman DN, Agrawal D, Leder K. The effect of age on immunologic response to recombinant hepatitis $B$ vaccine: a metaanalysis. Clin Infect Dis 2002;35:1368-1375.

7. Gisbert JP, Chaparro M, Esteve M. Review article: prevention and management of hepatitis B and C infection in patients with inflammatory bowel disease. Aliment Pharmacol Ther 2011;33:619-633.

8. Ray G. Current scenario of hepatitis B and its treatment in India. J Clin Transl Hepatol 2017;5:277-296.

9. Truelove SC, Witts LJ. Cortisone in ulcerative colitis; final re- port on a therapeutic trial. Br Med J 1955;2:1041-1048.

10. Mearin F, Lacy BE, Chang L, et al. Bowel disorders. Gastroenterology 2016;150:1393-1407.

11. WHO Publication. Hepatitis B vaccines: WHO position paper-recommendations. Vaccine 2010;28:589-590.

12. Chang JY, Jung SA, Moon CM, Kim SE, Jung HK, Shim KN. Response to hepatitis B vaccination in patients with inflammatory bowel disease: a prospective observational study in Korea. Intest Res 2018;16:599-608.

13. Jiang HY, Wang SY, Deng M, et al. Immune response to hepatitis B vaccination among people with inflammatory bowel diseases: a systematic review and meta-analysis. Vaccine 2017; 35:2633-2641.

14. Vida Pérez L, Gómez Camacho F, García Sánchez V, et al. Adequate rate of response to hepatitis $\mathrm{B}$ virus vaccination in patients with inflammatory bowel disease. Med Clin (Barc) 2009; 132:331-335.

15. Gisbert JP, Menchén L, García-Sánchez V, Marín I, Villagrasa JR, Chaparro M. Comparison of the effectiveness of two protocols for vaccination (standard and double dosage) against hepatitis B virus in patients with inflammatory bowel disease. Aliment Pharmacol Ther 2012;35:1379-1385.

16. Sempere L, Almenta I, Barrenengoa J, et al. Factors predicting response to hepatitis B vaccination in patients with inflammatory bowel disease. Vaccine 2013;31:3065-3071.

17. Andrade P, Santos-Antunes J, Rodrigues S, Lopes S, Macedo G. Treatment with infliximab or azathioprine negatively impact the efficacy of hepatitis B vaccine in inflammatory bowel disease patients. J Gastroenterol Hepatol 2015;30:1591-1595.

18. Belle A, Baumann C, Bigard MA, et al. Impact of immunosuppressive therapy on hepatitis B vaccination in inflammatory bowel diseases. Eur J Gastroenterol Hepatol 2015;27:877-881.

19. Cekic C, Aslan F, Kirci A, et al. Evaluation of factors associated with response to hepatitis $B$ vaccination in patients with inflammatory bowel disease. Medicine (Baltimore) 2015;94:e940.

20. Cossio-Gil Y, Martínez-Gómez X, Campins-Martí M, et al. Immunogenicity of hepatitis B vaccine in patients with inflammatory bowel disease and the benefits of revaccination. J Gastroenterol Hepatol 2015;30:92-98.

21. Etzion O, Novack V, Perl Y, et al. Sci-B-VacTM Vs ENGERIX-B vaccines for hepatitis B virus in patients with inflammatory bowel diseases: a randomised controlled trial. J Crohns Colitis 2016;10:905-912.

22. Pratt PK Jr, David N, Weber HC, et al. Antibody response to hepatitis B virus vaccine is impaired in patients with inflammatory bowel disease on infliximab therapy. Inflamm Bowel 
Dis 2018;24:380-386.

23. Nguyen DL, Nguyen ET, Bechtold ML. Effect of immunosuppressive therapies for the treatment of inflammatory bowel disease on response to routine vaccinations: a meta-analysis. Dig Dis Sci 2015;60:2446-2453.

24. Arora A, Singh SP, Kumar A, et al. INASL position statements on prevention, diagnosis and management of hepatitis B virus infection in India: the Andaman statements. J Clin Exp Hepatol 2018;8:58-80.
25. Sood A, Midha V, Sood N, Bhatia AS, Avasthi G. Incidence and prevalence of ulcerative colitis in Punjab, North India. Gut 2003;52:1587-1590.

26. Makharia GK, Ramakrishna BS, Abraham P, et al. Survey of inflammatory bowel diseases in India. Indian J Gastroenterol 2012;31:299-306.

27. World population review [Internet]. c2021 [cited 2019 Jun 1]. https://www.worldpopulationreview.com 